\title{
Resequencing of 388 cassava accessions identifies valuable loci and selection for variation in heterozygosity
}

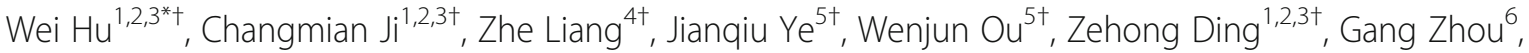 \\ Weiwei Tie ${ }^{1,2,3}$, Yan Yan ${ }^{1,2,3}$, Jinghao Yang ${ }^{2,3}$, Liming $\mathrm{Ma}^{6}$, Xiaoying Yang ${ }^{7}$, Yunxie Wei ${ }^{8}$, Zhiqiang Jin ${ }^{2,3}$, \\ Jianghui $X i e^{2,3}$, Ming Peng ${ }^{2,3}$, Wenquan Wang ${ }^{2,3}$, Anping Guo ${ }^{1,2,3}$, Biyu Xu $\mathrm{u}^{2,3}$, Jianchun Guo ${ }^{2,3}$, Songbi Chen ${ }^{5}$, \\ Mingcheng Wang ${ }^{6}$, Yang Zhou ${ }^{6}$, Xiaolong Li ${ }^{6}$, Ruoxi Li ${ }^{9}$, Xinhui Xiao ${ }^{5}$, Zhongqing Wan ${ }^{5}$, Feifei An ${ }^{5}$, Jie Zhang ${ }^{5}$, \\ Qingyun Leng ${ }^{5}$, Yin Li ${ }^{10}$, Haitao Shi ${ }^{*^{*}}$, Ray Ming ${ }^{11,12^{*}}$ (D) and Kaimian $\mathrm{Li}^{5^{*}}$
}

\footnotetext{
* Correspondence: huwei2010916@ 126.com; haitaoshi@hainanu.edu.cn; rayming@illinois.edu; likaimian@ sohu.com

'Wei Hu, Changmian Ji, Zhe Liang, Jianqiu Ye, Wenjun Ou and Zehong Ding contributed equally to this work.

'Sanya Research Institute of Chinese Academy of Tropical Agricultural Sciences, Sanya, Hainan, China ${ }^{8}$ Hainan Key Laboratory for Sustainable Utilization of Tropical Bioresources, College of Tropical Crops, Hainan University, Haikou, Hainan China

${ }^{11}$ FAFU and UIUC-SIB Joint Center for Genomics and Biotechnology, Fujian Provincial Key Laboratory of Haixia Applied Plant Systems Biology, Key Laboratory of Genetics, Breeding and Multiple Utilization of Crops, Ministry of Education, Fujian Agriculture and Forestry University, Fuzhou, China

${ }^{5}$ Tropical Crops Genetic Resources Institute, Chinese Academy of Tropical Agricultural Sciences, Haikou, Hainan, China Full list of author information is available at the end of the article
}

\begin{abstract}
Background: Heterozygous genomes are widespread in outcrossing and clonally propagated crops. However, the variation in heterozygosity underlying key agronomic traits and crop domestication remains largely unknown. Cassava is a staple crop in Africa and other tropical regions and has a highly heterozygous genome.

Results: We describe a genomic variation map from 388 resequenced genomes of cassava cultivars and wild accessions. We identify 52 loci for 23 agronomic traits through a genome-wide association study. Eighteen allelic variations in heterozygosity for nine candidate genes are significantly associated with seven key agronomic traits. We detect 81 selective sweeps with decreasing heterozygosity and nucleotide diversity, harboring 548 genes, which are enriched in multiple biological processes including growth, development, hormone metabolisms and responses, and immune-related processes. Artificial selection for decreased heterozygosity has contributed to the domestication of the large starchy storage root of cassava. Selection for homozygous GG allele in MeTIR1 during domestication contributes to increased starch content. Selection of homozygous AA allele in MeAHL17 is associated with increased storage root weight and cassava bacterial blight (CBB) susceptibility. We have verified the positive roles of MeTIR1 in increasing starch content and MeAHL17 in resistance to CBB by transient overexpression and silencing analysis. The allelic combinations in MeTIR1 and MeAHL17 may result in high starch content and resistance to CBB.
\end{abstract}

Conclusions: This study provides insights into allelic variation in heterozygosity associated with key agronomic traits and cassava domestication. It also offers valuable resources for the improvement of cassava and other highly heterozygous crops.

Keywords: Resequencing, Cassava, Heterozygosity, Selection, Agronomic traits 


\section{Background}

The capacity and efficiency of plant breeding contribute greatly to global food production and human life [1]. The development and application of genomic technologies offer powerful tools for precise selection and directional breeding [2]. Associations between genotypes and phenotypes in populations have revealed homozygous allelic variations that are significantly associated with key agronomic traits in many crops, including rice [3], maize [4], tomato [2], and cotton [5], thereby accelerating the breeding process. However, many crops are highly heterozygous due to outcrossing or clonal propagation, such as cassava [6], rubber tree [7], and mango [8]. Currently, the effect of variation in heterozygosity on agronomic traits is still less known.

Throughout the history of crop domestication, numerous wild species were domesticated into cultivated crops by artificial selection. Subsequently, along with human migration, directional selection, and further trait improvement to meet human demands, cultivated crops acquired region-specific differences in traits [2]. Using genome sequencing technologies, we can track crop domestication and breeding history, and thereby better understand how human selection shaped crop genomes, as shown in tomato [2], cotton [9], rice [10], maize [11], soybean [12], peach [13], melon [14], watermelon [15], and pineapple [16]. However, the variation in heterozygosity underlying crop domestication remains largely unknown.

Cassava (Manihot esculenta Crantz) belongs to the Euphorbiaceae family, and represents one of the most widely cultivated crops in tropical areas [6, 17]. Annual production of cassava is 276.7 million tons, and it provides staple food for nearly one billion people in 105 countries in tropical regions across Africa, South America, and Asia $[18,19]$. Cultivated cassava was domesticated from its wild progenitor, Manihot esculenta ssp. flabellifolia, in the Amazon basin over 6000 years ago [6, 20]. Domestication of cassava has resulted in characteristics including an annualized growth cycle, high initial growth rate, increased yield, and high starch content [20-24], which makes cassava a desirable energy source both for human consumption and industrial biofuel applications. Although cassava contributes greatly to food security in tropical areas and developing countries, research on cassava genomics and breeding is relatively lagging behind due to the limited genomic resources $[6,20,24,25]$. The development of population genomic resources will accelerate the progress of genetic improvement for this important crop.

The cassava genome $(2 \mathrm{n}=36)$ has a relatively high heterozygosity of $0.61-0.84 \%$ among cultivars and wild progenitors, which makes it an excellent system for studying variation in heterozygosity $[6,20,26]$. In the present study, we performed large-scale resequencing of cassava genomes, population genomic analyses, and genome-wide association studies (GWAS) for key agronomic traits. We identified valuable loci with variation in heterozygosity associated with key agronomic traits and our results offered insights into variation in heterozygosity during cassava domestication.

\section{Results}

Genome variation map and phylogenetic relationship

A total of 388 cassava accessions, including 14 wild progenitors, 38 landraces, and 336

breeding lines were collected to construct a cassava variation map. These samples 
include 51 accessions that were previously resequenced [6, 24] and 337 accessions that were resequenced in the present study, representing accessions from the main cassava production areas of 15 countries worldwide (Fig. 1a). A total of $3.35 \mathrm{~Tb}$ data was generated with an average sequencing depth of $9.45 \times$ of the reference genome SC205 [26] (Additional file 1: Table S1). We identified a total of 1,344,463 high-quality single-
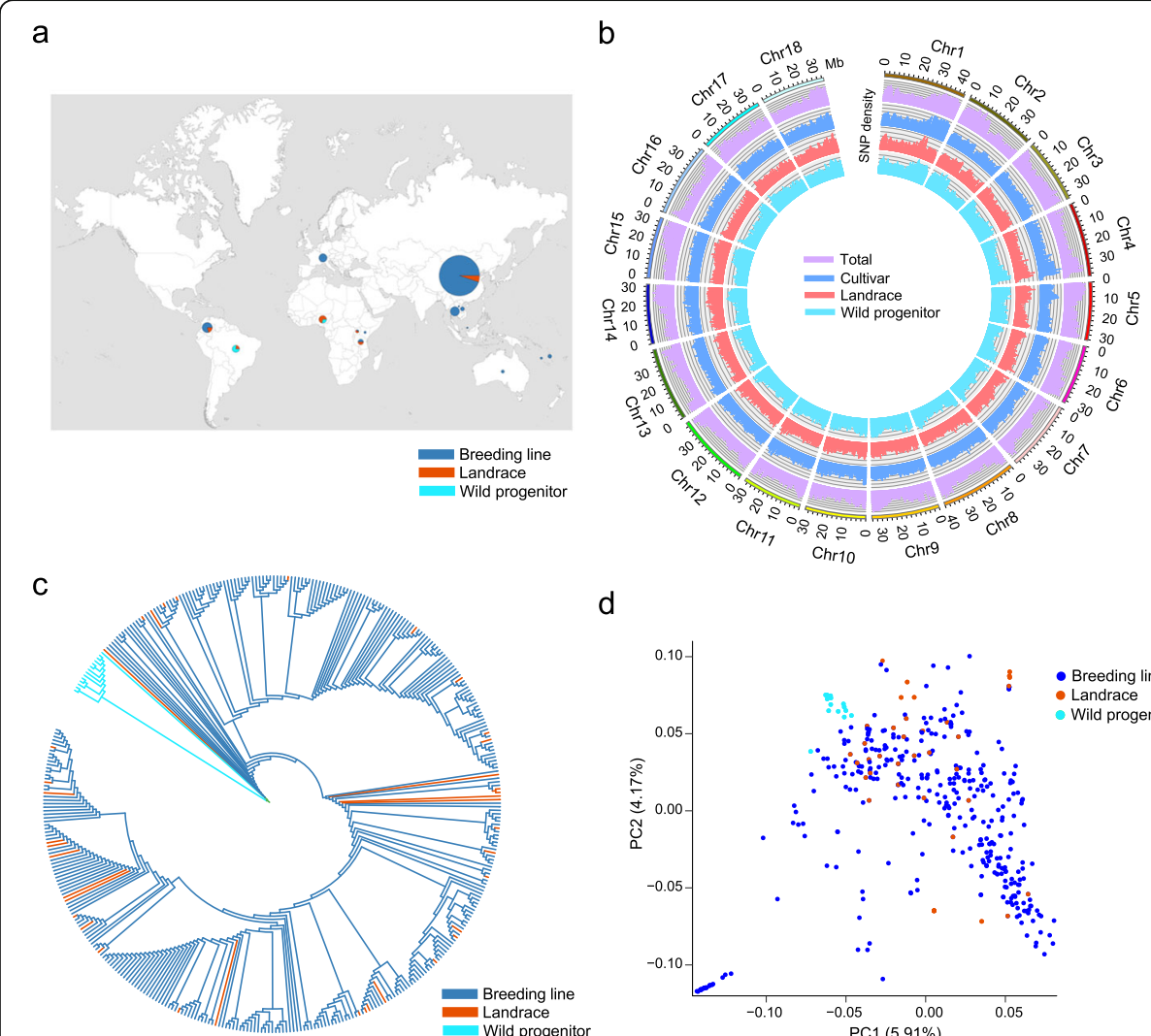

d
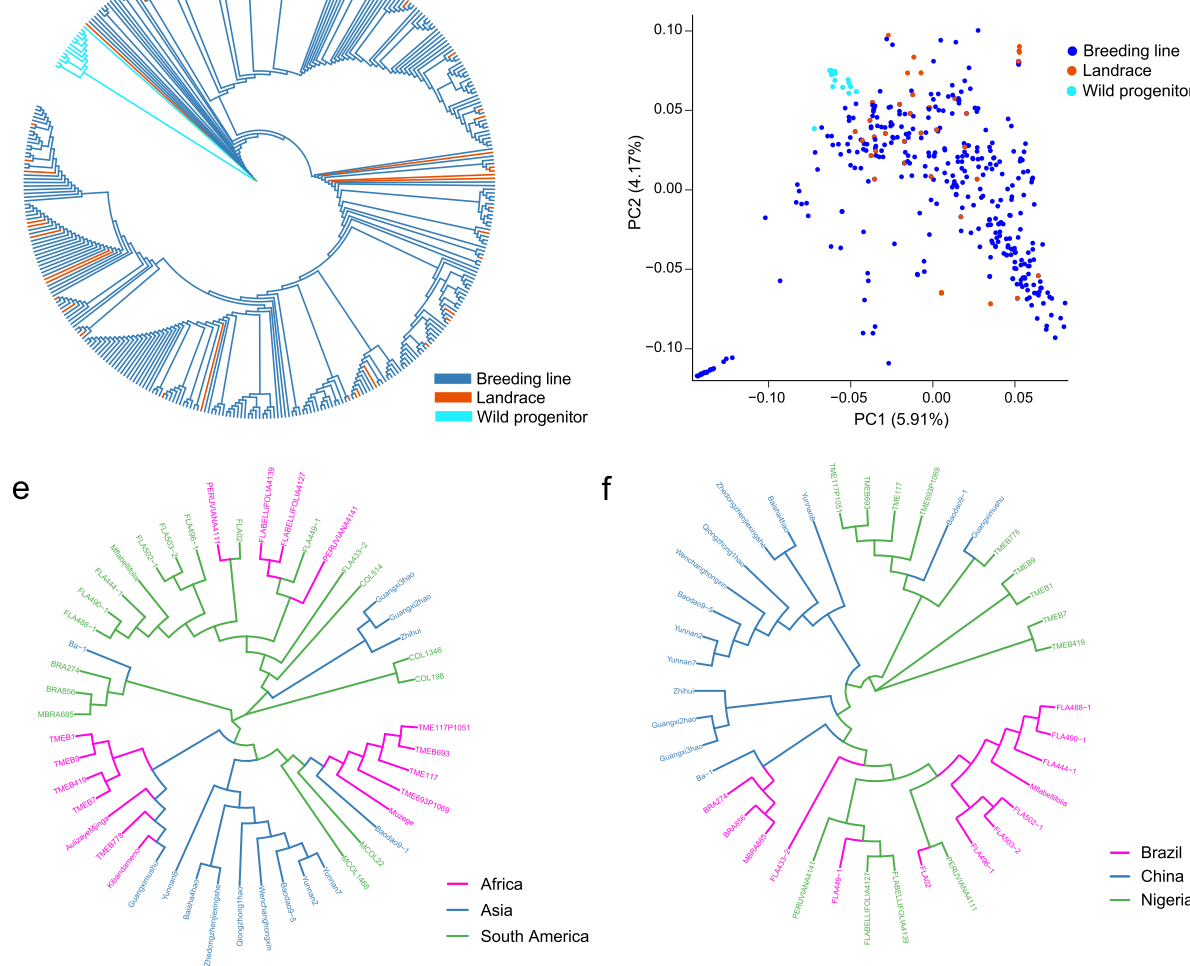

f

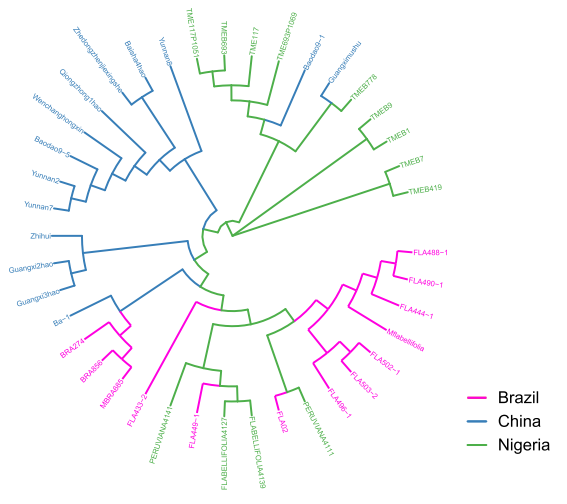

Fig. 1 Genome-wide variation map and phylogenetic relationship of cassava. a Geographic distribution of resequenced cassava accessions. The area of the circle is proportional to the number of cassava accessions. b SNP density distributions in 10-kb non-overlapping sliding windows of 388 resequenced cassava accessions. c Phylogeny of 388 cassava accessions generated using the neighbor-joining tree method with genome-wide SNPs. $\mathbf{d}$ Principal component analysis of 388 cassava accessions using genome-wide SNPs. PC1, first principal component; PC2, second principal component. e,f Phylogenic relationships of wild progenitors and landraces from different continents $(\mathbf{e})$ and countries $(\mathbf{f})$ analyzed using the neighborjoining tree method with genome-wide SNPS 
nucleotide polymorphisms (SNPs) and 1,018,832 InDels (shorter than $50 \mathrm{bp}$ ) (Fig. 1b, Additional file 2: Table S2). We observed that the average accuracy of the SNP calling is $94.2 \%$ by PCR-based sequencing (Additional file 3: Table S3). Phylogenetic and principal component analyses (PCA) classified the 388 cassava accessions into two major groups (Fig. 1c,d). Group I includes 13 wild progenitors and group II contains all of the landraces and breeding lines and a single wild progenitor (FLA433-2) belonging to $M$. esculenta ssp. flabellifolia (Additional file 22: Fig. S1). Notably, the wild progenitor FLA433-2 from Brazil shows cassava-like storage roots and is genetically admixed with the cultivars [6]. Its close phylogenetic relationship with all of the cultivars supports the hypothesis of the origin of cassava in South America with M. esculenta ssp. flabellifolia representing the most likely progenitor species of cultivated cassava [6, 17, 24]. The LD extent in cassava was lower in the wild progenitors $\left(7 \mathrm{~kb} ; r^{2}=0.2\right)$ than in the landraces $\left(85 \mathrm{~kb} ; r^{2}=0.2\right)$ and breeding lines (177 kb; $\left.r^{2}=0.2\right)$ (Additional file 22: Fig. S2).

We assessed genetic distances and phylogenetic relationship among populations of landraces and wild progenitors (Additional file 4: Table S4). On a continent scale, the values of $F_{\mathrm{ST}}$ and $p$-distance between cassava populations from South America and Africa $(0.071 ; 0.189)$ and between populations from Africa and Asia $(0.067 ; 1.017)$ were lower than those between populations from South America and Asia (0.094; 0.200). Cassava accessions from Africa clustered closely with the accessions from South America or Asia (Fig. 1e). On a country scale, the values of $F_{\mathrm{ST}}$ and $p$-distance between cassava populations from Brazil and Nigeria $(0.120 ; 0.174)$ and between populations from Nigeria and China $(0.088 ; 0.112)$ were lower than those between populations from Brazil and China $(0.149 ; 0.189)$. Cassava accessions from Nigeria clustered closely with the accessions from Brazil or China (Fig. 1f). These results indicate a close phylogenetic relationship between cassava populations from South America and Africa as well as between populations from Africa and Asia. This is in accordance with archeological evidence showing that cassava was present in South America more than 4000 years ago, following which it was taken from Brazil to the Atlantic coast of Africa in the 1500s after Europeans arrived on the American continent, and then to Southeast Asia in the 1600 s $[27,28]$.

\section{Genome-wide association study}

Based on 1,313,775 high-quality SNPs from 337 cassava accessions resequenced in the present study, we performed a GWAS analysis for 33 morphological traits during 2013, 2016, and 2020 (Additional file 5: Table S5). Finally, we identified 52 marker-trait associations (MTAs) for 23 agronomically important traits (Additional file 6: Table S6, Additional file 22: Fig. S3). Of which, seven traits were detected MTAs in at least 2 years, and two MTAs for stem height and storage root (SR) number per plant were repeatedly observed during 2016 and 2020 (Additional file 7: Table S7, Additional file 22: Fig. S4). In addition, two MTAs for SR weight and SR number per plant overlapped with four known quantitative trait loci (QTLs) [29, 30] (Additional file 8: Table S8).

Epidermal type (smooth or rough) and scar seriously affect the appearance quality of the SR. A significant signal on chromosome 10 associated with SR epidermal type was located at downstream of Sc10g012040 and upstream of Sc10g012050 (Fig. 2a,b), which encodes two auxin response factors that are crucial for various plant growth and development 


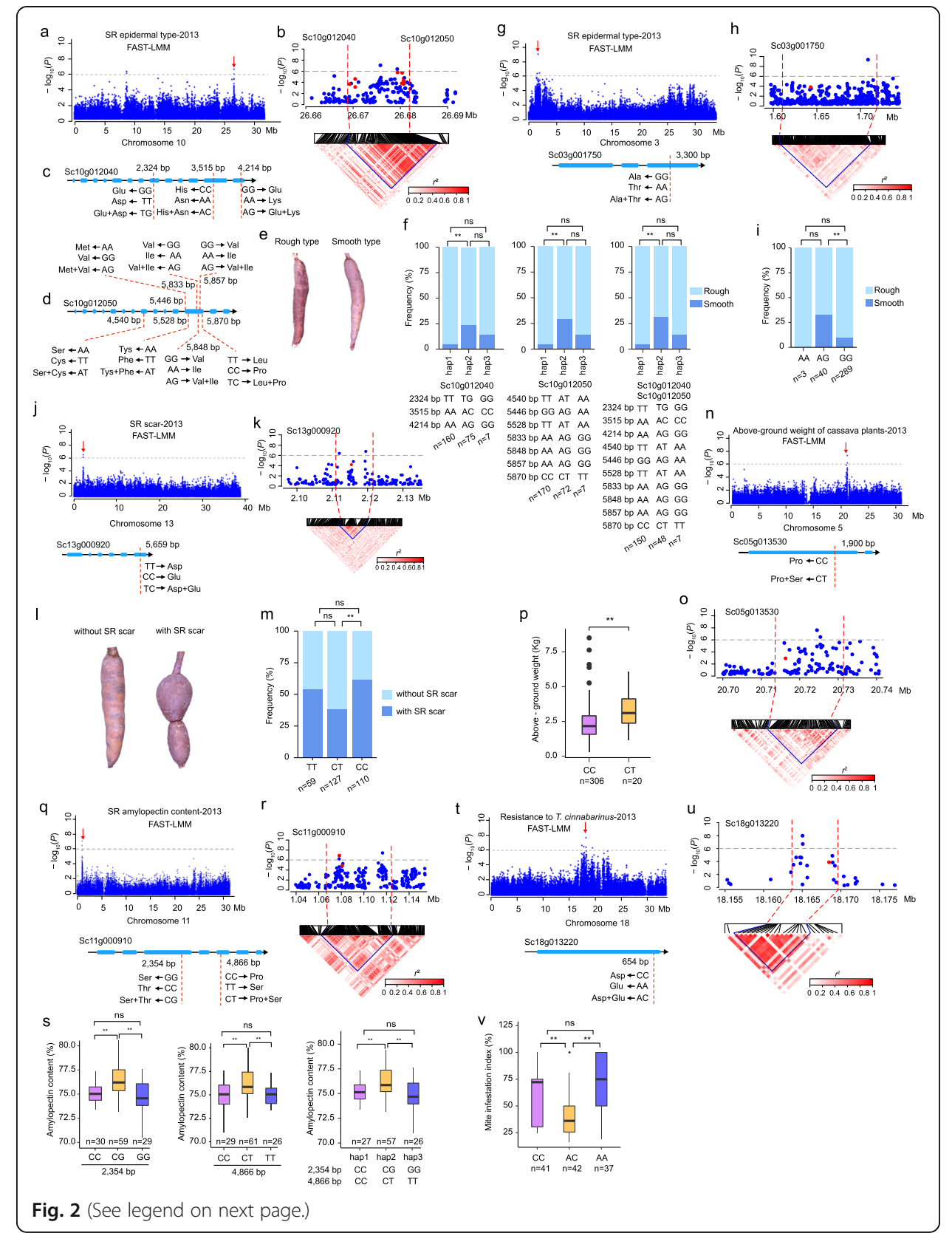


(See figure on previous page.)

Fig. 2 GWAS identification of candidate genes for variation in heterozygosity associated with key agronomic traits. a-f GWAS identification of Sc10g012040 and Sc10g012050 as candidate genes for SR epidermal type on chromosome 10. a Manhattan plots for SR epidermal type on chromosome 10 using FAST-LMM. Red arrow indicates the significant GWAS peak. b Local manhattan plot (top) and linkage disequilibrium heat map (bottom) surrounding the GWAS signal. Red dashed lines indicate the candidate region. Red dots indicate the core SNPs in the candidate gene. c,d Sc10g012040 and Sc10g012050 gene model, respectively. The dashed red lines represent the position of nonsynonymous SNPs. e Images of rough and smooth types of SR epidermis. $\mathbf{f}$ Comparison of SR epidermal types based on the haplotypes in Sc10g012040 (left) and Sc10g012050 (middle), as well as in both Sc10g012040 and Sc10g012050 (right). Hap 1 and hap 3 indicate the homozygous allele combinations, while hap 2 shows the heterozygous allele combinations. The symbol $n$ represents the number of accessions with the same genotype. $\mathbf{g}-\mathbf{i}$ GWAS identification of Sc03g001750 as a candidate gene for SR epidermal type on chromosome 3. j-m GWAS identification of Sc13g000920 as a candidate gene for SR scar on chromosome 13. $\mathbf{n}-\mathbf{p}$ GWAS identification of Sc05g013530 as a candidate gene for above-ground weight of cassava plants on chromosome 5. q-s GWAS identification of Sc11g000910 as a candidate gene for SR amylopectin content on chromosome 11. t-v GWAS identification of Sc18g013220 as a candidate gene for cassava resistance to T. cinnabarinus on chromosome 18. For box plots in (p), (s), and $(\mathbf{v})$, the center line represents the median, box limits indicate the upper and lower quartiles, and whiskers denote the range of the data. The significance of difference was derived with two-tailed $t$-test $\left({ }^{*} P<0.05,{ }^{*} P<0.01\right)$. For histograms in $(\mathbf{f}),(\mathbf{i})$, and $(\mathbf{m})$, the significance of difference was derived with the chi-square test $\left({ }^{*} P<0.05,{ }^{* *} P<0.01\right)$

processes [31]. We found that nonsynonymous SNPs, three in Sc10g012040 and seven in Sc10g012050, are associated with SR epidermal type (Fig. 2c,d). Cassava accessions carrying the heterozygous alleles and heterozygous haplotype within Sc10g012040 and Sc10g012050 showed higher frequency of smooth epidermis of SR than those carrying the homozygous alleles and homozygous haplotypes (Fig. 2e,f and Additional file 22: Fig. S5, S6). In addition, Sc03g001750 near another SR epidermal type signal on chromosome 3 encodes a mitochondrial carrier protein involved in plant development [32] (Fig. 2g,h). Sc03g001750 contains a nonsynonymous SNP (3300 bp) that is associated with SR epidermal type. The accessions carrying the heterozygous AG allele had higher frequency of smooth epidermis of SR than did those with the homozygous GG allele (Fig. 2i). Moreover, a SR scar signal on chromosome 13 was located at downstream of Sc13g000920 (Fig. 2j,k), which encodes an isopentenyl-diphosphate delta-isomerase that is essential for cytokinin biosynthesis [33]. A nonsynonymous SNP (5659 bp) in Sc13g000920 is associated with SR scar. The accessions harboring the heterozygous CT allele showed lower frequency of SR with scar than those harboring the homozygous CC allele (Fig. 2l,m).

The weight of the above-ground part of cassava reflects the growth vigor of the plant and becomes an important factor in its use as feed. A significant signal associated with aboveground weight of cassava plants on chromosome 5 harbors Sc05g013530 (Fig. 2n,o), which encodes a FAR1-related sequence protein that is known to regulate plant growth and development [34]. A nonsynonymous SNP (1900 bp) in Sc05g013530 has two types of allelic variation (CC and CT). The accessions harboring the heterozygous CT allele showed significantly higher above-ground weight than did those harboring the homozygous CC allele (Fig. 2p).

SR amylopectin content determines the industrial value of cassava. Sc11g000910 near a SR amylopectin content signal on chromosome 11 encodes a cytochrome P450 protein that is associated with starch accumulation during plant development [35] (Fig. 2q,r). We identified two nonsynonymous SNPs (2354bp and $4866 \mathrm{bp}$ ) in Sc11g000910, which is associated with SR amylopectin content. Cassava accessions carrying the heterozygous alleles and heterozygous haplotype had higher SR amylopectin content than those carrying the homozygous alleles and homozygous haplotypes (Fig. 2s). 
Storage roots with red endothelium may have high nutritional value and are popular among consumers. A strong peak on chromosome 2 associated with SR endothelial color harbors Sc02g008280, which encodes a glycosyltransferase that is required for anthocyanin biosynthesis [36] (Additional file 22: Fig. S7a,b). We found that a nonsynonymous SNP (522 bp) in Sc02g008280 was associated with SR endothelial color. The accessions harboring the AT or TT allele had higher frequency of SR with red endothelium than those harboring the AA allele (Additional file 22: Fig. S7c,d,e).

Susceptibility to mites, especially for Tetranychus cinnabarinus, bring about up to 50 70\% reduction in the production of cassava [37]. A peak associated with cassava resistance to $T$. cinnabarinus on chromosome 18 was located at the upstream of Sc18g013220 (Fig. 2t,u), which encodes a heat-shock protein that is widely involved in response to biotic and abiotic stresses [38, 39]. A nonsynonymous SNP (654 bp) in Sc18g013220 is associated with cassava resistance to T. cinnabarinus. The accessions harboring the heterozygous AC allele showed significantly lower mite infestation index than did those harboring the homozygous CC or AA allele (Fig. 2v).

We examined the expression levels of these candidate genes and found that all these genes expressed abundantly in different tissues or stages of SR development (Additional file 9: Table S9, Additional file 22: Fig. S8). Together with the above allelic variation analysis, these results suggest the potential roles of allelic variation in heterozygosity on key agronomic traits, of which cassava accessions carrying heterozygous alleles in each of these candidate gene had more desirable phenotype than did those carrying the corresponding homozygous alleles.

\section{Identification of highly heterozygous blocks}

It is necessary to retain the heterozygous loci that control excellent traits during breeding. We identified 1395 heterozygous blocks (occupying $27.9 \mathrm{Mb}$ ) with high frequency (above the $95 \%$ threshold) in cultivars, defined as highly heterozygous blocks, involving 3718 genes (Additional file 10: Table S10, Additional file 22: Fig. S9a). Kyoto Encyclopedia of Genes and Genomes (KEGG) analysis suggested that "starch and sucrose metabolism" is the most significantly enriched group for these genes (Additional file 11: Table S11, Additional file 22: Fig. S9h). We characterized 79 highly heterozygous blocks that are associated with starch accumulation in the storage root (Additional file 12: Table S12). For example, Sc02g005160 (encoding a glycogen phosphorylase) and Sc02g005330 (encoding an alphaamylase), which are involved in starch metabolism [40, 41], were located on the blocks w17969 and w17979, respectively. We further observed 20 highly heterozygous blocks that overlapped with 6 GWAS signals (Additional file 13: Table S13, Additional file 22: Fig. S9). Moreover, cassava accessions carrying the highly heterozygous block on chromosome 1 showed higher frequency of red endothelium of SR than those carrying the corresponding homozygous block (Additional file 22: Fig. S9i). These results further support the hypothesis that variation in heterozygosity is associated with key agronomic traits.

\section{Selection signatures of heterozygosity in cassava}

To trace the possible evolutionary scenario of heterozygosity, we split the cassava genome into heterozygous and homozygous regions according to heterozygous SNPs of the reference gnome. We then compared the genomic diversity between heterozygous 
and homozygous regions in 374 cultivars. The heterozygous regions possessed significantly higher genetic diversity ( $\pi$ value) and positive Tajima's $D$ value than the homozygous regions in most (16 out of 18) chromosomes, suggesting that balancing selection has contributed to the maintenance of genomic heterozygosity. This finding is also supported by the lower $F_{\mathrm{ST}}$ value in heterozygous regions than in homozygous regions in most (14 out of 18) chromosomes between cultivars and wild progenitors (Fig. 3a-c, Additional file 14: Table S14).

Artificial selection is of great importance for domestication of crops for desirable phenotypic traits [42]. To identify potential signals of selection related to heterozygosity during cassava domestication, we scanned genomic regions that exhibit drastic decreases in heterozygosity above the $99 \%$ threshold by comparing wild progenitors and cultivars (including landraces and breeding lines) and identified 140 selective sweeps that cover $1.65 \%(11.7 \mathrm{Mb})$ of the assembled genome and harbor 941 protein-coding genes (Fig. 3d, Additional file 15: Table S15). We also identified 138 selective sweeps (above the 99\% threshold) that show reduction in nucleotide diversity by comparing wild progenitors and cultivars, covering $11.6 \mathrm{Mb}$ of the assembled genome and harboring 851 protein-coding genes (Fig. 3d, Additional file 16: Table S16). We found 81 selective sweeps with both decreases in heterozygosity and nucleotide diversity, covering 6.5 Mb of the assembled genome and harboring 548 protein-coding genes (Fig. 3d, Additional file 17: Table S17). These genes were enriched in multiple biological processes including growth, development, hormone metabolisms and responses, and immune-related processes (Additional file 18: Table S18).

\section{Selection for decreases in heterozygosity contributes to domestication of the large starchy storage root in cassava}

Domestication has led to significant increases in yield and starch content of cassava storage roots. Storage root weight and starch content in cultivars are about 60 and 3 times higher than in wild progenitors, respectively (Fig. 4a, b). To understand the mechanism underlying this phenomenon, we identified growth- and developmentassociated genes that are differentially expressed in storage roots between wild progenitors and cultivars within selective sweep regions with both decreases in heterozygosity and nucleotide diversity, including MeTIR1 (Sc02g014200) and MeAHL17 (Sc02g014000) (Fig. 3d, Fig. 4c, and Additional file 19: Table S19).

MeTIR1 is an ortholog of TIR1 in Arabidopsis, which encodes a transport inhibitor response 1 protein and functions as the long-sought auxin receptor [43]. Auxin biosynthesis and TIR1-mediated auxin signaling are required for starch synthesis [44, 45]. We found a selection signal for decreased heterozygosity around MeTIR1 on chromosome 2 (Fig. 4d). Expression of MeTIR1 was significantly higher in cultivars compared to wild progenitors (Fig. 4c, e). We found that a SNP in the upstream region (- $5067 \mathrm{bp}$ ) of MeTIR1 might be associated with MeTIR1 expression and domestication (Fig. 4f). Compared with wild progenitors, cultivars showed a lower frequency of the CG and CC alleles, but a higher frequency of the homozygous GG allele (Fig. 4g). Moreover, starch content was significantly higher in accessions carrying the GG allele than in accessions carrying the CG allele (Fig. 4h). Accordingly, MeTIR1 exhibited significantly higher expression in accessions carrying the GG allele than in accessions carrying the CG and CC alleles (Fig. 4i). We further 


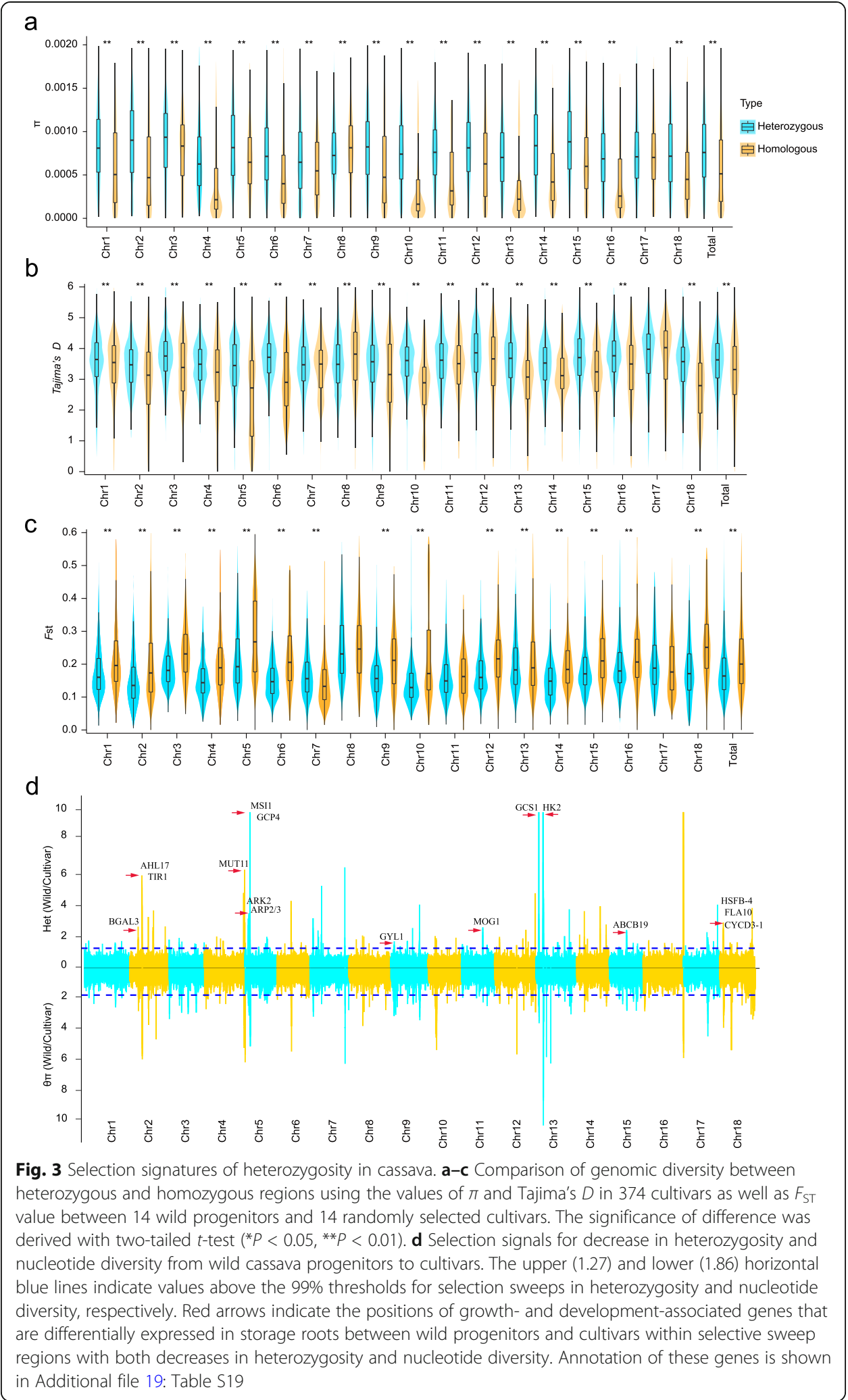




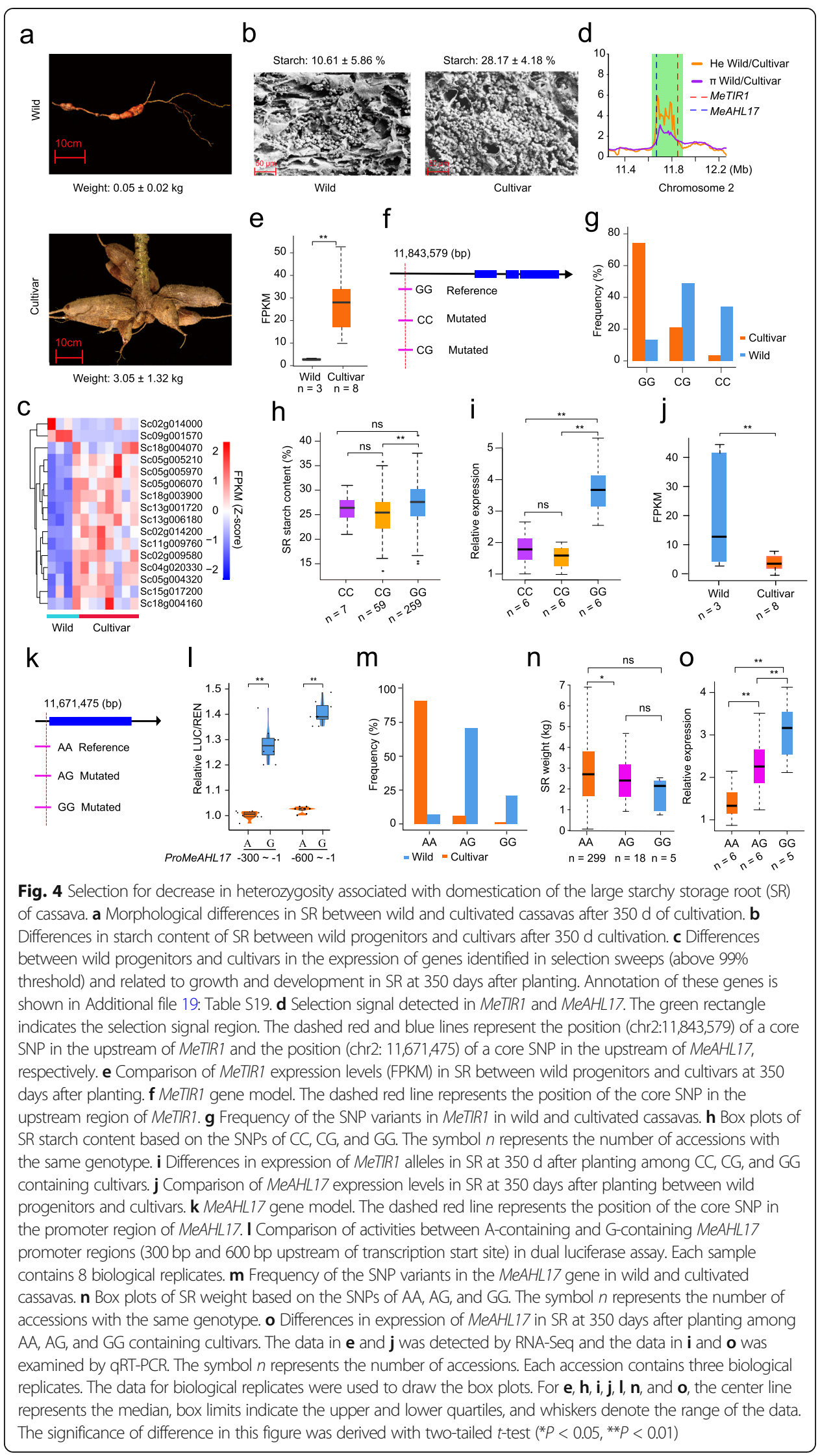


validated the function of MeTIR1 in cassava using transient overexpression and silencing technology and found that overexpression of MeTIR1 in F1015 (GG allele), R72 (GG allele), 4363 (CG allele), and Baodao9-1 (CG allele) significantly increased starch content, whereas silencing of MeTIR1 in F1015 (GG allele) and R72 (GG allele) led to decreased starch content in cassava leaves (Additional file 22: Fig. S10).

In addition, we found a strong selection signal for decreased heterozygosity around MeAHL17 on chromosome 2 (Fig. 4d). MeAHL17, an ortholog of AHL17 in Arabidopsis, encodes an AT-hook motif nuclear-localized transcription factor that is associated with inhibiting diverse developmental processes [46-48]. Expression of MeAHL17 was significantly lower in cultivars than in wild progenitors (Fig. 4c, j). Further, we found that a SNP in the upstream region (-53 bp) of MeAHL17 might be associated with MeAHL17 expression and domestication (Fig. 4k). The dual luciferase assay indicated that the promoter regions of MeAHL17 carrying $\mathrm{G}$ had higher activity than those carrying A, suggesting that this allelic variation affects MeAHL17 expression (Fig. 4l). Compared with wild progenitors, cultivars showed a lower frequency of AG and GG alleles, but a higher frequency of the homozygous AA allele (Fig. 4m). Storage root weight was significantly higher in accessions carrying the AA allele than in accessions carrying the AG allele (Fig. 4n), which is significantly associated with lower MeAHL17 expression in accessions carrying AA than in those carrying AG (Fig. 4o).

\section{MeAHL17 is positively associated with cassava bacterial blight (CBB) susceptibility}

Cassava bacterial blight (CBB), caused by Xanthomonas axonopodis pv. manihotis (Xam), is one of the most severe threats to cassava production [49]. We found a strong association signal on chromosome 2 with a $-\log _{10} P$ value of 7.97 (Fig. 5a). We focused on the locus mapped from 11.65 to $11.70 \mathrm{Mb}$ with six candidate genes (Sc02g013980 through Sc02g014030) (Fig. 5b), of which only MeAHL17 showed significant induction after Xam inoculation (Additional file 20: Table S20). In chickpea, an AHL member was mapped to be associated with Ascochyta blight resistance [50]. We found that the same allelic SNP variation as shown in Fig. 4k in the promoter region of MeAHL17 is associated with $\mathrm{CBB}$ resistance. The accessions carrying the AA allele of MeAHL17 showed significantly higher frequency of susceptibility to $\mathrm{CBB}$ than those carrying the AG allele (Fig. 5c). Although the transcripts of MeAHL17 in all 12 accessions were largely induced after Xam infection at 2 days post inoculation (dpi), the transcripts of MeAHL17 in CBB-susceptible accessions (carrying the AA allele) were significantly lower than those in CBB-resistant accessions (carrying the AG allele) at both $0 \mathrm{dpi}$ and 2 dpi (Fig. 5d). Moreover, overexpression of MeAHL17 in Yunnan8 (with AG allele), ZM9781 (with AG allele), ZM95308 (with AA allele), and RuishiX3 (with AA allele) increased CBB resistance by leaf bacterial populations, whereas silencing of MeAHL17 in Yunnan8 (with AG allele) and ZM9781 (with AG allele) increased CBB susceptibility (Fig. 5e, f and Additional file 22: Fig. S11). These results suggest that MeAHL17 is positively associated with $\mathrm{CBB}$ resistance.

\section{Combinations of allelic variation in MeTIR1 and MeAHL17}

MeAHL17 and MeTIR1 are located within the same selective sweep region on chromosome 2 (Figs. 4d and 6a). We performed a linkage disequilibrium analysis of MeAHL17 


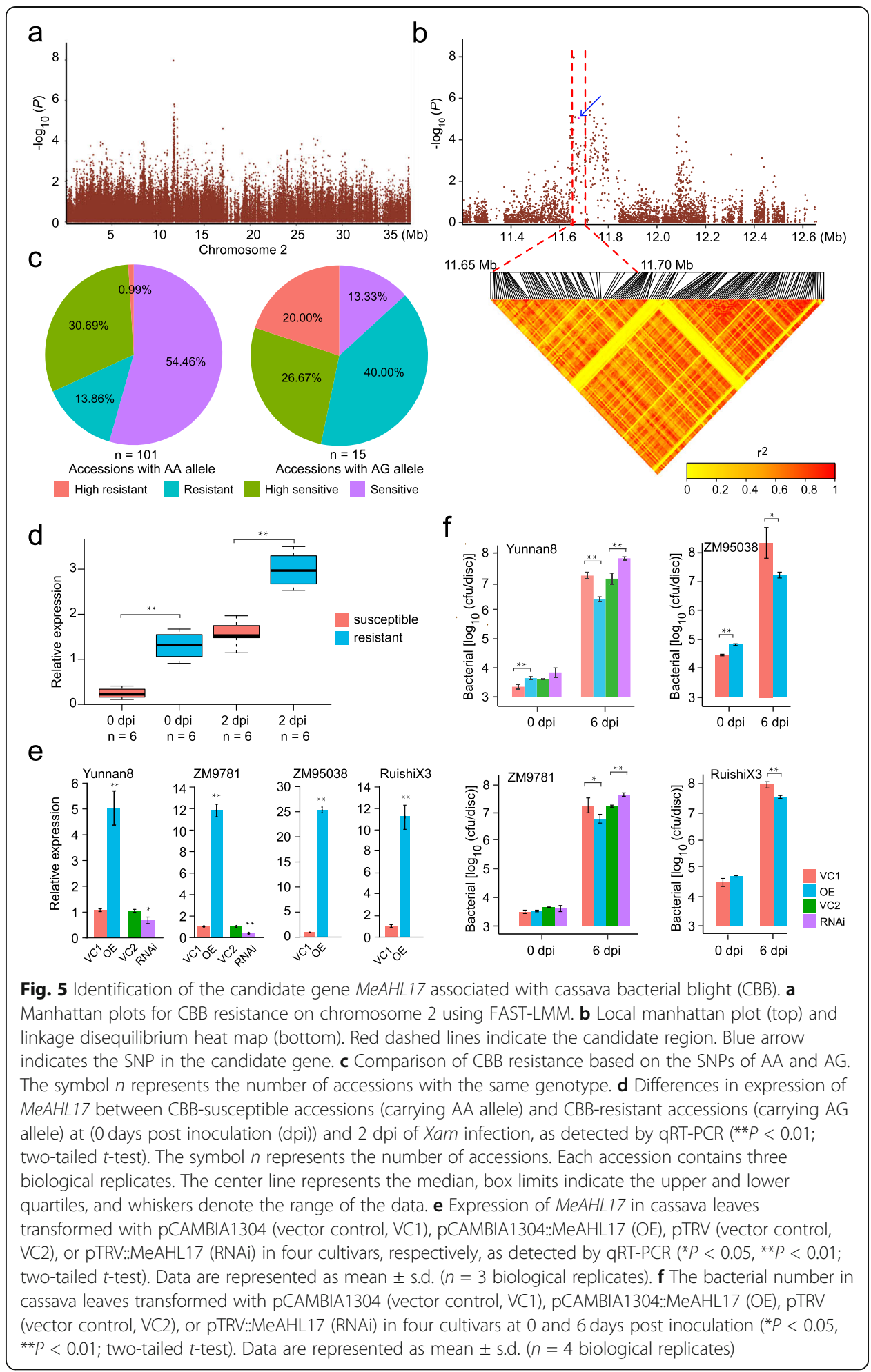

and MeTIR1 loci and found that they are tightly linked (Fig. 6b). The frequency of allelic combinations of AA (MeAHL17) and GG (MeTIR1) as well as AA (MeAHL17) and CG (MeTIR1) significantly increased during domestication (Fig. 6v). However, the starch content and the frequency of resistance to $\mathrm{CBB}$ were not the most desirable in 


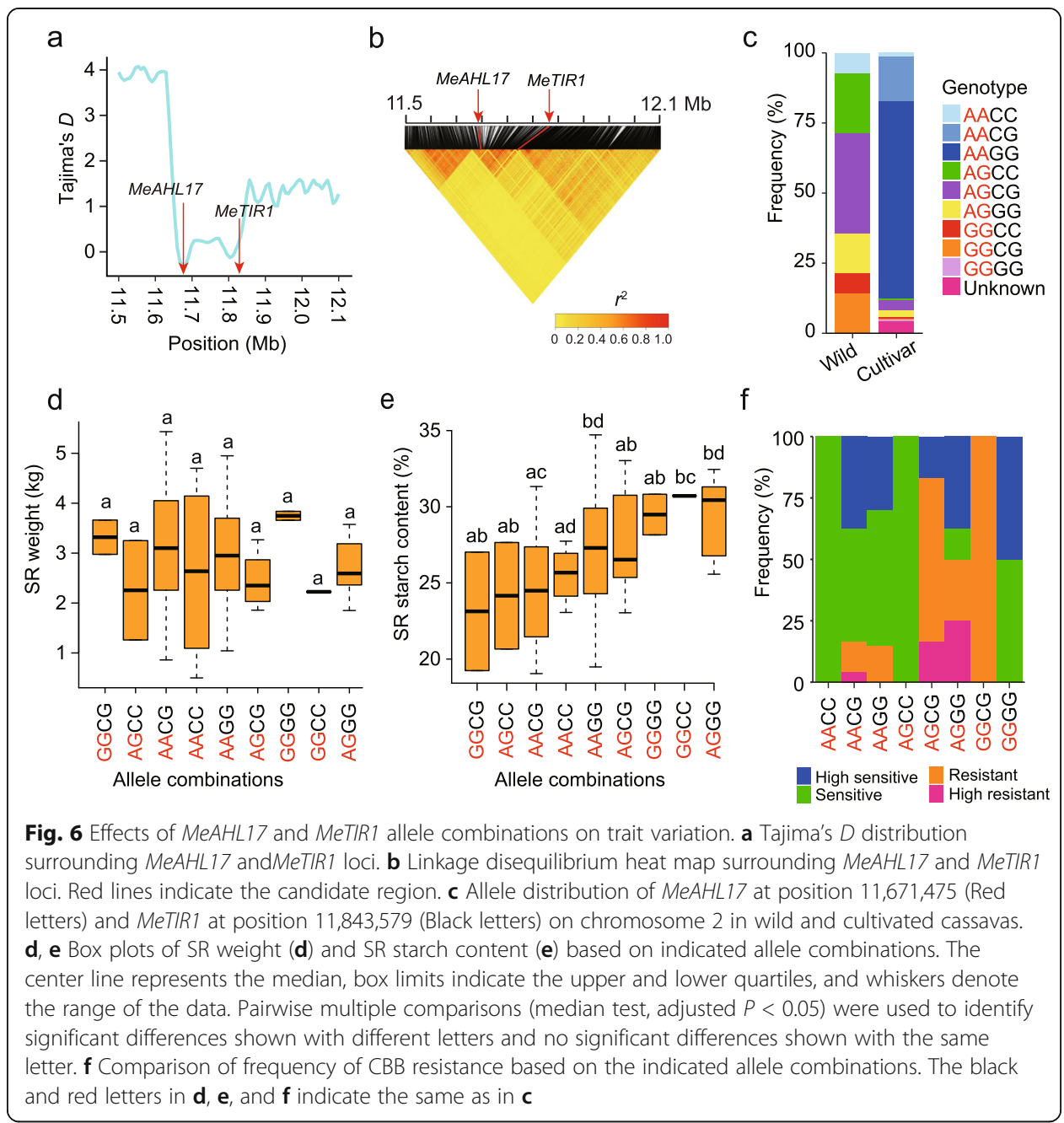

cultivars carrying the two allelic combinations (Fig. 6d, e, f). We also found that cultivars carrying the combinations of AG (MeAHL17) and GG (MeTIR1) alleles showed high starch content and high frequency of resistance to CBB (Fig. 6e, f).

\section{Discussion}

Generation of heterozygous genomes by hybridization between or within species can help maintain plant diversity and serve as a potential source of new species [51]. Cassava is monoecious with unisexual flowers, which lead to out crossing that increase genomic heterozygosity. Extensive hybridization and clonal reproduction yield and maintain the high heterozygosity of cassava [6]. Balancing selection has contributed to the maintenance of cassava heterozygosity, which is in accordance with the role of balancing selection on retaining genetic diversity [52-54]. In contrast, comparison of wild accessions and cultivars revealed 81 selective sweeps with both decreases in heterozygosity and nucleotide diversity, suggesting that decrease in nucleotide diversity by artificial selection is accompanied with reduction in heterozygosity during cassava domestication. Modern cassava breeding has primarily focused on yield and starch content of storage roots [24]. Decreases in both heterozygosity and nucleotide diversity of MeTIR1 and MeAHL17 by artificial selection contributed to the domestication of the 
large starchy storage root. However, the selection of the homozygous genotype of MeAHL17 led to the adverse effect of CBB susceptibility. The allele combinations of MeTIR1 and MeAHL17 resulting from variation in heterozygosity may confer high starch content and resistance to $\mathrm{CBB}$, which will be beneficial for future cassava breeding.

Artificial selection has shaped crop genomes to meet human demands and has also resulted in the near fixation of a large proportion of the genome $[2,55]$. There is an urgent need to utilize genetic diversity in germplasm resources for crop improvement [56]. Effective screening of non-selected regions could provide favorable alleles to use for retaining genetic diversity and future breeding. Several promising candidate genes that were found to be highly associated with key traits of cassava from GWAS analysis are in non-selected regions (Fig. 2). Cassava accessions carrying the heterozygous alleles in each candidate gene showed more desirable phenotype than did those carrying the corresponding homozygous alleles. Additionally, we characterized 79 highly heterozygous blocks associated with starch accumulation as well as a highly heterozygous block associated with red endothelium of storage root. Retaining of these heterozygous alleles and blocks during future breeding may be beneficial for excellent agronomic traits and genetic diversity of cassava. This deepens our understanding of variation in heterozygosity affecting agronomic traits and provides potential targets for selection during cassava breeding.

The availability of genomic resources is not in itself sufficient to increase crop productivity [56]. It is important for crop improvement to systematically evaluate germplasm resources and access allelic variations affecting agronomic traits. With the release of the cassava genome, the research on population genetics of cassava reveals several fundamental issues in cassava biology, especially the nature of its extensive interspecific hybridization and fixation of deleterious mutations during clonal propagation [6, 24]. Currently, the use of large-scale resequencing data to precisely identify valuable loci associated with key agronomic traits of cassava remains poorly understood. Resequencing 388 cassava accessions yielded 1,344,463 high-quality SNPs. These comprehensive data and the high-density genetic markers offer adequate coverage to investigate association between markers and causal loci controlling agronomic traits, as previously reported in rice [3], cotton [5], tomato [2], and soybean, etc [12]. We detected 52 loci associated with 23 agronomic traits of cassava by GWAS analysis with multiple statistical models. Of which, the allelic variation in heterozygosity of nine candidate genes associated with key agronomic traits were identified and the orthologs of several candidate genes, such as Sc10g012040, Sc10g012050, Sc13g000920, and Sc02g014000, have been functionally characterized in other species. In addition, it is possible to identify association between allelic variation of homozygous genotypes and agronomic traits from significant GWAS signals. The data obtained from resequencing and GWAS will facilitate future functional genomic studies and designing breeding strategies in cassava.

Taken together, this study identified valuable loci with variation in heterozygosity associated with key agronomic traits and offered insights into variation in heterozygosity during cassava domestication, which provides a large amount of new genomic resources for utilization of heterozygosity to accelerate cassava improvement. Further work will be required to validate candidate genes underlying the key traits because of 
the limited phenotyping replicates for GWAS analysis. In addition, more wild progenitors should be collected and resequenced to further verify the accuracy of our domestication analysis due to the limited number of wild progenitors used in this study.

\section{Conclusions}

This study provided a variation map of 388 cassava accessions, identified 52 loci for 23 agronomic traits, and revealed allelic variation in heterozygosity associated with key agronomic traits and cassava domestication. We found that artificial selection of homozygous alleles in MeTIR1 and MeAHL17 contributes to the domestication of the large starchy storage root. Notably, selection of homozygous alleles in MeAHL17 is associated with increased both storage root weight and CBB susceptibility. Our findings will contribute to elucidating the genetic basis of the variation in heterozygosity associated with key agronomic traits and cassava domestication as well as allow for the strategic exploitation for cassava improvement. This should facilitate breeding programs, not only in cassava but also in other highly heterozygous crops.

\section{Methods}

\section{Sampling and whole-genome resequencing}

A total of 337 cassava accessions, including 20 landraces and 317 breeding lines, were collected from the Chinese Cassava Germplasm Bank (Danzhou, China) for wholegenome resequencing. The plants grew in the planting base at the Tropical Crops Genetic Resources Institute of Chinese Academy of Tropical Agricultural Sciences. Fresh young leaves were frozen in liquid nitrogen for genomic DNA extraction using a DNeasy Plant Mini Kit (Qiagen, Beijing, China). Paired-end libraries with insert size of $500 \mathrm{bp}$ were constructed with $5 \mu \mathrm{g}$ of genomic DNA for each sample. The 150-bp paired-end reads for each sample were sequenced using the Illumina X-Ten platform. The raw data of genome resequencing was deposited in the Sequence Read Archive under a NCBI BioProject accession PRJNA578024 [57]. The other 38 cassava accessions previously resequenced by Bredeson et al. [6] and 13 accessions previously resequenced by Ramu et al. [24] were downloaded from NCBI-SRA (https://www.ncbi.nlm.nih.gov/) and Cassava Base (ftp://ftp.cassavabase.org/HapMapII/), respectively.

\section{Reads alignment, variation calling, and SNP validation}

The sequencing reads for each sample were mapped to the cassava SC205 reference genome in the BWA mem v0.7.17 [58] program with the default parameters. The mapping results were processed by sorting and duplicate marking using Samtools v1.9 [59] and Picard v1.94 (https://broadinstitute.github.io/picard/). After removing the low mapping quality $(\mathrm{MQ}<20)$ reads, both single-end and paired-end mapped reads were used to detect SNPs in the GATK toolkitv3.5 [60] process. Mapped reads resulting from PCR duplicates were removed. The HaplotypeCaller module was used to establish a raw population genotype file containing the SNPs and indels that was further filtered using the parameters: "QUAL $<2.0||$ QD $<2.0||$ MQ $<40.0||$ FS $>60.0||$ MQRankSum $<-12.5||$ ReadPosRankSum $<-8.0$-clusterSize 2 -clusterWindowSize 5" and "QD $<2.0$ || FS > 200.0 || ReadPosRankSum <-20.0." The annotation of the identified SNPs and indels were performed using SnpEff v3.6c tool software [61]. A total of 26 SNPs 
were randomly selected for PCR-based sequencing from 33 accessions. All PCR products were aligned against the target region of SC205 genome using Sequencher 5.4, and the genotypes were manually checked for each accession.

\section{Phylogenetic analysis}

After filtering the low-quality SNPs using metrics of "minor allele frequency (MAF) > 0.05 and integrity $>0.8$ " from the entire SNP dataset, we obtained a subset of $1,344,463$ high confidence SNPs for phylogenetic analysis. To investigate the phylogenetic relationships of the 388 cassava accessions, an unrooted phylogenetic tree was constructed using the neighbor-joining method with the Kimura 2-parameter model and 1000 bootstrap replicates in MEGA5.10 [62]. The consensus trees were exhibited with the online Interactive tree of life (iTOL) v3 (https://itol.embl.de) [63].

\section{Principal component analysis and linkage disequilibrium analysis}

Principal component analysis (PCA) was carried out using the smartPCA program from the EIGENSOFT package v.6.0.1 (https://github.com/DReichLab/EIG) with 1,344,463 high confidence SNPs. The first two principal components were used to separate the cultivar and wild progenitor samples. Linkage disequilibrium between pairs of SNPs with MAF $>0.05$ and integrity $>0.8$ was assessed as the correlation coefficient $\left(r^{2}\right)$ using PLINKv1.07 with the parameters (--maf 0.05 --r2 --ld-window 999999 --ld-window-kb 1000 --ld-window-r20) [64]. LD decay was estimated based on $r^{2}$ between two SNPs and averaged in 1-kb windows with a maximum distance of $1 \mathrm{Mb}$.

\section{$F$-statistic and $p$-distance calculation}

The fixation statistic $F_{\mathrm{ST}}$ and $p$-distance were used to estimate the degree of pairwise genomic differentiation between pairs of populations, based on the variance in allele frequencies. The $F_{\mathrm{ST}}$ values between different geographical groups were evaluated using PopGenome package v2.7.1 (https://popgenome.weebly.com/). The $p$-distance values between different groups were estimated using MEGA X with the $p$-distance model [65].

\section{Phenotyping}

A total of 337 accessions were planted in Danzhou, Hainan Province (109.5 E, 19.5 N). The cassava plants were planted in early March 2013/2016 and harvested in late February 2014/2017, as well as planted in early May 2020 and harvested in early May 2021. Each accession was grown in one row that consisted of eight plants with $0.8 \mathrm{~m}$ distance between each plant. Phenotyping of whole plant-, stem-, storage root-, postharvest-, and resistance-related traits was performed according to the Chinese description specification for cassava germplasm resources (NY1943-2010) in 2013, 2016 and 2020. The phenotypic data of plant height, biomass, stem diameter, stem height, aboveground weight, SR weight, SR length, and SR number for each accession were defined as the average of the eight plants. The SR starch content, SR starch viscosity, SR starch gelatinization temperature, and SR amylopectin content were examined according to previous methods [66]. The data for each accession were defined as the mean of three biological replicates. Resistance to T. cinnabarinus and Xam of cassava accessions was evaluated following the Chinese technical specification for evaluating cassava resistance 
to pests (NY/T 2445-2013) and bacterial blight (NY/T 3005-2016). The mean value from three biological replicates was employed to determine the resistance levels.

\section{Genome-wide association study}

In total, 1,313,775 high-quality SNPs with a minor allele frequency (MAF) $>0.05$ and integrity $>0.8$ in the 337 cassava cultivars were used for GWAS of 33 traits. The GWAS analyses were performed using different statistical models of generalized linear model (GLM), mixed linear model (MLM), compressed mixed linear model (CMLM), efficient mixed model association expedited (EMMAX), and Factored Spectrally Transformed Linear Mixed Models (FAST-LMM). The Q matrix was generated by ADMIXTURE v1.3.0 [67] as fixed effects. In the MLM and CMLM model, the kinship (K) matrix was constructed using SPAGeDi v1.3a [68]. The $P$ value threshold of 0.000001 was set to control the genome-wide type I error rate [69]. MTAs supported at least by two statistical models are defined as valid MTAs. Genes located in 100-kb windows which centered on the MTAs were considered as potential candidate genes for further analysis. The potential candidate genes that have annotation and transcripts were selected for further analyzing whether they are in the LD block region and have allelic variation associated with phenotypic differences.

\section{RNA sequencing and expression quantification}

Three biological replicates were included in each RNA-Seq experiment. The root, stem, leaf samples, and the storage root at seven development stages from 100 to 340 DAP (days after planting) were collected from SC205 for RNA-Seq experiments. We also sampled the storage roots from three wild progenitors and eight cultivars for transcriptome sequencing. The RNAs were isolated using the RNeasy Plus Mini kit DP441 (Qiagen, Beijing, China), and cDNA libraries were prepared using an NEBNext Ultra RNA Library Prep Kit. The quantified libraries were then prepared for sequencing using the Illumina HiSeq X-Ten platform. Adapter sequences and low-quality reads (more than 20\% low-quality bases) were removed. The clean reads were mapped to the SC205 reference genome by HISAT2v2.0.4 [70]. The gene expression levels were calculated by StringTie v1.3.4d [71] using the default parameters. The gene expression level was normalized by the fragments per kilobase of transcript per million mapped reads (FPKM). The expressed genes were defined as FPKM values greater than 0.1. Differentially expressed genes (DEGs) were identified using the DEseq2 package [72]. Significant differences in expression levels were tested using a two-tailed $t$-test. The DEGs were defined as a fold change (FC) larger than 2 or smaller than 0.5 and a false discovery rate $(F D R)<0.01$. The raw data of transcriptomic sequencing was deposited in the Sequence Read Archive under a NCBI BioProject accession PRJNA578024 [57].

\section{Identification of heterozygous blocks with high frequency in cultivars}

The heterozygosity of each $20 \mathrm{~kb}$ non-overlapping window was calculated as "heterozygosity SNPs / window length" for each cultivar accession. The average heterozygosity of the windows was used as a cutoff value to distinguish heterozygous or homologous windows. The window that has more heterozygous sites than the average heterozygous SNPs (10.83 heterozygous SNPs per window) was considered as a heterozygous block 
in corresponding accession, while the contrary was regarded as a homologous block. We calculated the population frequency of the heterozygous blocks in 374 cultivars to identify the highly heterozygous blocks. The sliding windows within the empirical top $5 \%$ of heterozygous frequency in the population were selected as highly heterozygous blocks. For each highly heterozygous block region, we separated the 374 cultivars into heterozygous and homologous groups according their heterozygosity information. We then detected trait segregation using trait values between heterozygous and homologous groups in highly heterozygous blocks using the Wilcoxon rank-sum test method. The genes in the highly heterozygous blocks associated with excellent traits were manually investigated according the functional description of their orthologs in the Arabidopsis database.

\section{Genetic diversity analysis between heterozygous and homozygous regions}

We remapped Illumina short reads of SC205 to its own reference genome and called SNPs using BWA mem v0.7.17 [58] and GATK toolkitv3.5 [60]. The rate of heterozygous SNPs for each window was calculated by sliding windows as formula: heterozygous SNPs / window length $\times 100 \%$ (window $=25 \mathrm{~kb}$ and step $=5 \mathrm{~kb}$ ). The window that has the rate of heterozygous SNPs less than 0.002 is considered as homozygous region; otherwise, it is considered as heterozygous region. The adjacent homozygous or heterozygous windows were merged into one large region, respectively. Only the regions whose length are more than $50 \mathrm{~kb}$ were used for genetic diversity analysis. We compared the genomic diversity between heterozygous and homozygous regions using the values of $\pi$ and Tajima's $D$ in 374 cultivars as well as $F_{\mathrm{ST}}$ value between 14 wild progenitors and 14 randomly selected cultivars at the genome and chromosome levels. The $\pi$, Tajima's $D$, and $F_{\mathrm{ST}}$ values in each chromosome were calculated using PopGenome package v2.7.1 [73] (https://popgenome.weebly.com/) for 50-kb sliding windows with a $10-\mathrm{kb}$ step size. The two-tailed $t$-test was used to examine the significant differences of genetic diversity between heterozygous and homozygous regions.

\section{Genome-wide selective sweep analysis}

All of the SNPs with a minor allele frequency (MAF) $>0.05$ and integrity $>0.8$ were selected for selective sweep analysis. Expected heterozygosity $(\mathrm{He})$ and nucleotide diversity $(\pi)$ analyses were applied to estimate the degree of variability based on the variance in allele frequencies of wild progenitors and cultivars (including landraces and breeding lines). The expected heterozygosity $(\mathrm{He})$ of each polymorphism site was calculated as $1-\Sigma P_{i}^{2}$, where $P_{i}$ represents the frequency of the $i$ allele. To detect the selective signals, the average He of each window was calculated for $50-\mathrm{kb}$ sliding windows with a 10-kb step size along each chromosome. Additionally, $\pi$ was calculated using PopGenome packagev2.7.1 [73] (https:// popgenome.weebly.com/) for 50-kb sliding windows with a 10-kb step size along each chromosome. Sliding windows within the empirical top $1 \%$ of He (wild/cultivar) or $\pi$ (wild/ cultivar) values for each comparison were identified as the candidate selective sweep regions, respectively. Adjacent confident windows were merged into a single selective sweep region.

\section{Functional characterization of MeTIR1 and MeAHL17 in cassava}

To explore the effect of the allele variation (at - 53 bp upstream of MeAHL17) on the activity of MeAHL17 promoter, we performed a dual luciferase assay in cassava 
protoplasts. The promoter sequences (from 0 to $-300 \mathrm{bp}$ and from 0 to $-600 \mathrm{bp}$ ) of MeAHL17 carrying G or A were cloned and inserted into the pGreenII0800-LUC vector. The recombinant constructs were transformed into cassava protoplasts using previous described method [74]. We examined the relative luciferase activity using a Dual Luciferase Reporter Gene Assay Kit (RG027, Beyotime, Shanghai, China). To detect the expression profiles of MeAHL17 in response to Xam, cassava leaves were collected at different time points. Total RNA was isolated and reverse transcribed using a cDNA Synthesis Kit (K1622, Thermo Scientific, Waltham, MA, USA). Then, the relative expression level of MeAHL17 was qualified by quantitative real-time PCR (qRT-PCR) using the $2^{-\triangle \Delta \mathrm{Ct}}$ method with MeEF1a as an internal reference. To overexpress MeTIR1 or MeAHL17, the coding sequences of MeTIR1 and MeAHL17 were amplified from SC205 and inserted into the pCAMBIA1304 vector, respectively. An Agrobacterium tumefaciens strain (GV3101) that harbors the recombinant constructs or pCAMBIA1304 was syringe infiltrated into cassava leaves as previously described [75]. To silence MeTIR1 or MeAHL17, MeTIR1- and MeAHL17-specific regions were amplified and cloned into the pTRV2 vector. An Agrobacterium tumefaciens strain (GV3101) that harbors the recombinant construct or PTRV2, together with pTRV1, were syringe infiltrated into cassava leaves as previously described [751]. After 2 days of cultivation for pCAMBIA1304::MeTIR1 and pCAMBIA1304 transformed plants and 2 weeks of cultivation for pTRV::MeTIR1 and pTRV transformed plants, we collected the cassava leaves to examine gene expression levels by qRT-PCR and starch content using a Starch Assay Kit (A148-1-1, Jiancheng, Nanjing, China). After 2 days of cultivation for pCAMBIA1304::MeAHL17 and pCAMBIA1304 transformed plants and 2 weeks of cultivation for pTRV::MeAHL17 and pTRV transformed plants, Xam was inoculated into the cassava leaves for 6 days, following which we examined the bacterial number and gene expression levels. The primer pairs used for qRT-PCR analysis are shown in Additional file 21: Table S21.

\section{Supplementary Information}

The online version contains supplementary material available at https://doi.org/10.1186/s13059-021-02524-7.

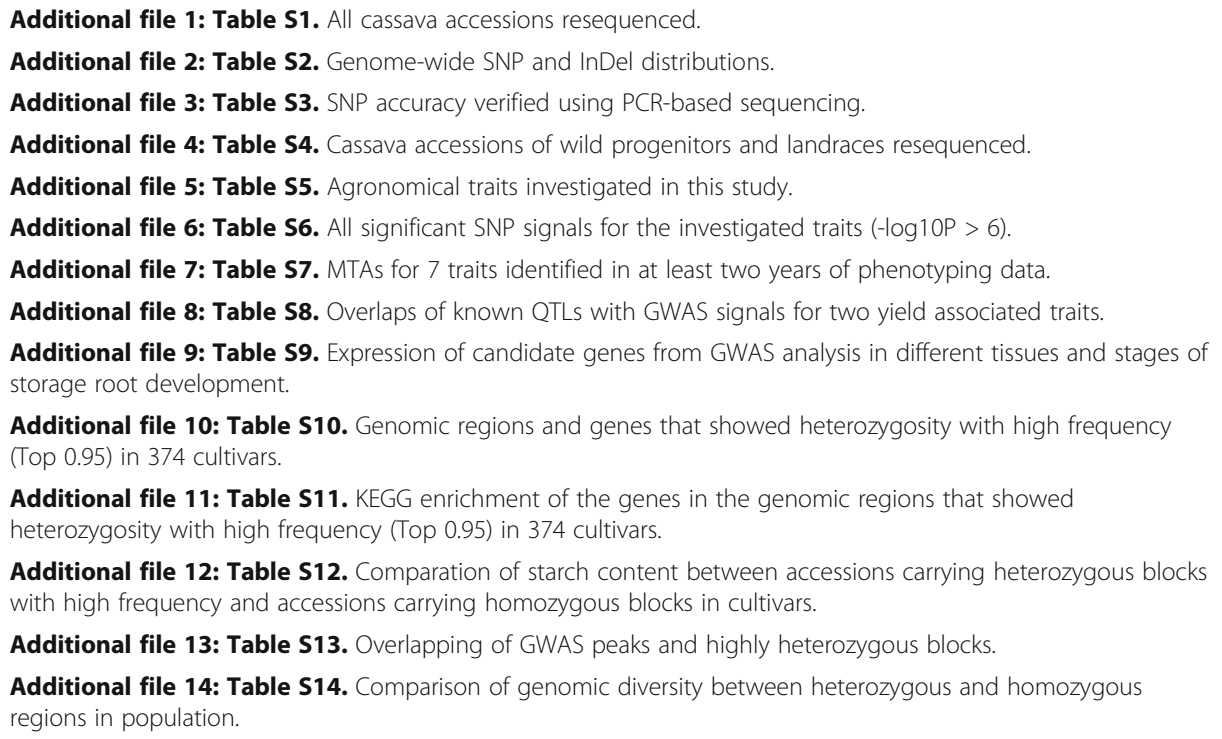


Additional file 15: Table S15. Genomic regions and genes that exhibit a decrease in heterozygosity during domestication from wild progenitors to cultivars (Top 0.99).

Additional file 16: Table S16. Genomic regions and genes that exhibit a decrease in nucleotide diversity during domestication from wild progenitors to cultivars (Top 0.99).

Additional file 17: Table S17. Genomic regions and genes that exhibit a decrease in both heterozygosity and nucleotide diversity during domestication from wild progenitors to cultivars (Top 0.99).

Additional file 18: Table S18. GO enrichment of the genes that exhibit a decrease in both heterozygosity and nucleotide diversity during domestication from wild progenitors to cultivars (Top 0.99).

Additional file 19: Table S19. Expression of the genes related to growth and development within selection sweeps with both decreases in heterozygosity and nucleotide (above $99 \%$ threshold) during cassava domestication.

Additional file 20: Table S20. Expression levels of the six genes after Xam inoculation within the locus mapped from 11.65 to $11.70 \mathrm{Mb}$.

Additional file 21: Table S21. Primer pairs used for GRT-PCR.

Additional file 22: Fig. S1-S11. Fig. S1. Phylogeny of 388 cassava accessions generated using the neighborjoining tree method with genome-wide SNPs. Fig S2. Linkage disequilibrium (LD) decay for different groups. Fig. S3. Manhattan plots for GWAS analysis of cassava agronomic traits. Fig. S4. Manhattan plots of two repeatedly observed MTAs for stem height and storage root number per plant. Fig S5. Comparison of SR epidermal types based on the non-synonymous SNPs in Sc10g012040. Fig. S6. Comparison of SR epidermal types based on the nonsynonymous SNPs in Sc10g012050. Fig. S7. GWAS identification of Sc02g008280 as a candidate gene for SR endothelial color on chromosome 2. Fig. S8. Expression of candidate genes from GWAS analysis in different tissues and stages of storage root development.Fig. S9. Identification and screening of heterozygous blocks with high frequency in cultivars. Fig. S10. Transient overexpression and silencing of MeTIR1 affect starch content in leaves of four cassava cultivars (F1015, R72, 4363 and Baodao9-1).Fig. S11. Photos of cassava leaves transformed with pCAMBIA1304 (vector control, VC1), pCAMBIA1304::MeAHL17 (OE), pTRV (vector control, VC2) or pTRV::MeAHL17 (RNAi) in four cultivars at 0 and 6 days post inoculation.

Additional file 23. Review history.

\section{Acknowledgements}

We thank X.H. Huang (Shanghai Normal University), F. Cheng (Chinese Academy of Agricultural Sciences), and X.T. Zhang (Fujian Agriculture and Forestry University) for helpful discussions. We also thank Guangxi Subtropical Crops Research Institute for supplying materials.

\section{Review history}

The review history is available as Additional file 23.

\section{Peer review information}

Wenjing She was the primary editor of this article and managed its editorial process and peer review in collaboration with the rest of the editorial team.

\section{Authors' contributions}

W.H., R.M., and K.L. designed and managed the project. K.L., J.Y., W.O., Z.D., W.T., Y.Y., and JH.Y. collected the samples. L.M., M.W., Y.Z., and X.L. performed the genome resequencing. W.H., C.J., Z.L., H.S., Z.D., G.Z., X.Y., Y.W., R.L., and Q.L. performed data analyses. J.Y., W.O. X.X., Z.W., F.A., and J.Z. performed traits investigation and analyses. H.S. W.H., Z.J., J.X., M.P., W.W., A.G., B.X., J.G., S.C., and Y.W. designed and performed the molecular experiments. W.H. and C.J. wrote the manuscript. Z.L., R.M., and Y.L. revised the manuscript. All authors read and approved the final manuscript.

\section{Funding}

This work was supported by the National Key Research and Development Program of China (grant no

2019YFD1001101 and 2019YFD1000500), the 2020 Research Program of Sanya Yazhou Bay Science and Technology City (SKJC-2020-2-002), the Central Public-Interest Scientific Institution Basal Research Fund for Chinese Academy of Tropical Agricultural Sciences (grant no. 1630012019009, 1630052016005, 1630052016006, 1630052017021 and 1630052019023), the Central Public-Interest Scientific Institution Basal Research Fund for Innovative Research Team Program of CATAS (grant no. 17CXTD-28 and 1630052017017), and the earmarked fund for Modern Agro-industry Technology Research System (grant no. nycytx-11).

\section{Availability of data and materials}

The raw sequencing data reported in this study has been deposited in the Sequence Read Archive (SRA) under a NCB BioProject accession (PRJNA578024) [57]. The resequencing and RNA-Seq data have been deposited under NCBI BioSample accessions (SRR10389908-SRR10390244 and SRR10480846-SRR10480905). The cassava reference genome used in this study has been deposited in the NCBI-SRA [26]. The published resequencing data used in this study is available from NCBI-SRA [6] and CassavaBase [24]. The known QTLs used in this study is available from NCBI-SRA [29, 30].

\section{Declarations}

Ethics approval and consent to participate

Not applicable 


\section{Consent for publication}

Not applicable

\section{Competing interests}

The authors declare that they have no competing interests.

\section{Author details}

${ }^{1}$ Sanya Research Institute of Chinese Academy of Tropical Agricultural Sciences, Sanya, Hainan, China. ${ }^{2}$ Hainan Key Laboratory for Biosafety Monitoring and Molecular Breeding in Off-Season Reproduction Regions, Key Laboratory of Biology and Genetic Resources of Tropical Crops, Institute of Tropical Bioscience and Biotechnology, Chinese Academy of Tropical Agricultural Sciences, Haikou, Hainan, China. ${ }^{3}$ Hainan Key Laboratory for Protection and Utilization of Tropical Bioresources, Hainan Institute for Tropical Agricultural Resources, Chinese Academy of Tropical Agricultural Sciences, Haikou, Hainan, China. ${ }^{4}$ Biotechnology Research Institute, Chinese Academy of Agricultural Sciences, Beijing, China. ${ }^{5}$ Tropical Crops Genetic Resources Institute, Chinese Academy of Tropical Agricultural Sciences, Haikou, Hainan, China. ${ }^{6}$ Biomarker Technologies Corporation, Beijing, China. ${ }^{7}$ College of Food Science and Technology, Qilu University of Technology (Shandong Academy of Sciences), Jinan, China. ${ }^{8}$ Hainan Key Laboratory for Sustainable Utilization of Tropical Bioresources, College of Tropical Crops, Hainan University, Haikou, Hainan, China. ${ }^{9}$ Fu Foundation School of Engineering and Applied Science, Columbia University, New York, NY 10027, USA. ${ }^{10}$ Waksman Institute of Microbiology, Rutgers, The State University of New Jersey, Piscataway, NJ, USA. ${ }^{11}$ FAFU and UIUC-SIB Joint Center for Genomics and Biotechnology, Fujian Provincial Key Laboratory of Haixia Applied Plant Systems Biology, Key Laboratory of Genetics, Breeding and Multiple Utilization of Crops, Ministry of Education, Fujian Agriculture and Forestry University, Fuzhou, China. ${ }^{12}$ Department of Plant Biology, University of Illinois at Urbana-Champaign, Urbana, IL, USA.

Received: 22 February 2021 Accepted: 24 October 2021

Published online: 16 November 2021

\section{References}

1. Borlaug NE. Contributions of conventional plant breeding to food production. Science. 1983;219(4585):689-93. https:// doi.org/10.1126/science.219.4585.689.

2. Lin T, Zhu G, Zhang J, Xu X, Yu Q, Zheng Z, et al. Genomic analyses provide insights into the history of tomato breeding. Nat Genet. 2014;46(11):1220-6. https://doi.org/10.1038/ng.3117.

3. Huang X, Wei X, Sang T, Zhao Q, Feng Q, Zhao Y, et al. Genome-wide association studies of 14 agronomic traits in rice landraces. Nat Genet. 2010;42(11):961-7. https://doi.org/10.1038/ng.695.

4. Li H, Peng Z, Yang X, Wang W, Fu J, Wang J, et al. Genome-wide association study dissects the genetic architecture of oil biosynthesis in maize kernels. Nat Genet. 2013;45(1):43-50. https://doi.org/10.1038/ng.2484.

5. Ma Z, He S, Wang X, Sun J, Zhang Y, Zhang G, et al. Resequencing a core collection of upland cotton identifies genomic variation and loci influencing fiber quality and yield. Nat Genet. 2018;50(6):803-13. https://doi.org/10.1038/s41 588-018-0119-7.

6. Bredeson JV, Lyons JB, Prochnik SE, Wu GA, Ha CM, Edsinger-Gonzales E, et al. Sequencing wild and cultivated cassava and related species reveals extensive interspecific hybridization and genetic diversity. Nat Biotechnol. 2016;34(5):562-70. https://doi.org/10.1038/nbt.3535.

7. Tang $C$, Yang M, Fang Y, Luo Y, Gao S, Xiao X, et al. The rubber tree genome reveals new insights into rubber production and species adaptation. Nat Plants. 2016;2(6):16073. https://doi.org/10.1038/nplants.2016.73.

8. Wang P, Luo Y, Huang J, Gao S, Zhu G, Dang Z, et al. The genome evolution and domestication of tropical fruit mango. Genome Biol. 2020;21(1):60. https://doi.org/10.1186/s13059-020-01959-8.

9. Wang $M$, Tu L, Lin M, Lin Z, Wang P, Yang Q, et al. Asymmetric subgenome selection and cis-regulatory divergence during cotton domestication. Nat Genet. 2017;49(4):579-87. https://doi.org/10.1038/ng.3807.

10. Huang $X$, Kurata $N$, Wei $X$, Wang $Z X$, Wang $A$, Zhao $Q$, et al. A map of rice genome variation reveals the origin of cultivated rice. Nature. 2012;490(7421):497-501. https://doi.org/10.1038/nature11532.

11. Hufford MB, Xu X, van Heerwaarden J, Pyhajarvi T, Chia JM, Cartwright RA, et al. Comparative population genomics of maize domestication and improvement. Nat Genet. 2012;44(7):808-11. https://doi.org/10.1038/ng.2309.

12. Zhou Z, Jiang Y, Wang Z, Gou Z, Lyu J, Li W, et al. Resequencing 302 wild and cultivated accessions identifies genes related to domestication and improvement in soybean. Nat Biotechnol. 2015;33(4):408-14. https://doi.org/1 $0.1038 / \mathrm{nbt} .3096$.

13. Li Y, Cao K, Zhu G, Fang W, Chen C, Wang X, et al. Genomic analyses of an extensive collection of wild and cultivated accessions provide new insights into peach breeding history. Genome Biol. 2019;20(1):36. https://doi.org/10.1186/s13 059-019-1648-9.

14. Zhao G, Lian Q, Zhang Z, Fu Q, He Y, Ma S, et al. A comprehensive genome variation map of melon identifies multiple domestication events and loci influencing agronomic traits. Nat Genet. 2019;51(11):1607-15. https://doi.org/10.1038/s41 588-019-0522-8.

15. Guo S, Zhao S, Sun H, Wang X, Wu S, Lin T, et al. Resequencing of 414 cultivated and wild watermelon accessions identifies selection for fruit quality traits. Nat Genet. 2019;51(11):1616-23. https://doi.org/10.1038/s41588-019-0518-4.

16. Chen $L Y$, VanBuren $R$, Paris M, Zhou H, Zhang $X$, Wai CM, et al. The bracteatus pineapple genome and domestication of clonally propagated crops. Nat Genet. 2019;51(10):1549-58. https://doi.org/10.1038/s41588-019-0506-8.

17. Olsen KM, Schaal BA. Evidence on the origin of cassava: phylogeography of Manihot esculenta. Proc Natl Acad Sci USA. 1999;96(10):5586-91. https://doi.org/10.1073/pnas.96.10.5586.

18. Li S, Cui Y, Zhou Y, Luo Z, Liu J, Zhao M. The industrial applications of cassava: current status, opportunities and prospects. J Sci Food Agric. 2017;97(8):2282-90. https://doi.org/10.1002/jsfa.8287.

19. Bull SE, Ndunguru J, Gruissem W, Beeching JR, Vanderschuren H. Cassava: constraints to production and the transfer of biotechnology to African laboratories. Plant Cell Rep. 2011;30(5):779-87. https://doi.org/10.1007/s00299-010-0986-6. 
20. Wang W, Feng B, Xiao J, Xia Z, Zhou X, Li P, et al. Cassava genome from a wild ancestor to cultivated varieties. Nat Commun. 2014;5(1):5110. https://doi.org/10.1038/ncomms6110.

21. Pujol B, Muhlen G, Garwood N, Horoszowski Y, Douzery EJ, McKey D. Evolution under domestication: contrasting functional morphology of seedlings in domesticated cassava and its closest wild relatives. New Phytol. 2005;166(1):30518. https://doi.org/10.1111/j.1469-8137.2004.01295.x.

22. An F, Chen T, Stephanie DM, Li K, Li QX, Carvalho LJ, et al. Domestication syndrome is investigated by proteomic analysis between cultivated cassava (Manihot esculenta Crantz) and its wild relatives. PLoS One. 2016;11(3):e0152154. https://doi.org/10.1371/journal.pone.0152154.

23. El-Sharkawy MA. Cassava biology and physiology. Plant Mol Biol. 2004;56(4):481-501. https://doi.org/10.1007/s11103005-2270-7.

24. Ramu P, Esuma W, Kawuki R, Rabbi IY, Egesi C, Bredeson JV, et al. Cassava haplotype map highlights fixation of deleterious mutations during clonal propagation. Nat Genet. 2017;49(6):959-63. https://doi.org/10.1038/ng.3845.

25. Rabbi IY, Udoh LI, Wolfe M, Parkes EY, Gedil MA, Dixon A, Ramu P, Jannink JL, Kulakow P. Genome-wide association mapping of correlated traits in cassava: dry matter and total carotenoid content. Plant Genome. 2017;10(3):1-14. https:// doi.org/10.3835/plantgenome2016.09.0094.

26. Hu W, Ji C, Shi H, Liang Z, Ding Z, Ye J, et al. Allele-defined genome reveals biallelic differentiation during cassava evolution. Mol Plant. 2021;14(6):851-4. https://doi.org/10.1016/.molp.2021.04.009.

27. Lebot V. Tropical root and tuber crops: cassava, sweet potato, yams and aroids. Wallingford: CABI Publishers; 2009.

28. Zhang J. A preliminary discussion on history of cassava's development. Agric Hist China. 2011;2:19-30.

29. Zou M, Lu C, Zhang S, Chen Q, Sun X, Ma P, et al. Epigenetic map and genetic map basis of complex traits in cassava population. Sci Rep. 2017;7(1):41232. https://doi.org/10.1038/srep41232.

30. Wang B, Guo X, Zhao P, Ruan M, Yu X, Zou L, et al. Molecular diversity analysis, drought related marker-traits association mapping and discovery of excellent alleles for 100-day old plants by EST-SSRs in cassava germplasms (Manihot esculenta Cranz). PLoS One. 2017;12(5):e0177456. https://doi.org/10.1371/journal.pone.0177456.

31. Lanctot A, Nemhauser JL. It's Morphin' time: how multiple signals converge on ARF transcription factors to direct development. Curr Opin Plant Biol. 2020;57:1-7. https://doi.org/10.1016/j.pbi.2020.04.008.

32. Xu J, Yang J, Wu Z, Liu H, Huang F, Wu Y, et al. Identification of a dual-targeted protein belonging to the mitochondrial carrier family that is required for early leaf development in rice. Plant Physiol. 2013;161(4):2036-48. https://doi.org/10.11 04/pp.112.210831.

33. Añorga M, Pintado A, Ramos C, De Diego N, Ugena L, Novák O, et al. Genes ptz and idi, coding for cytokinin biosynthesis enzymes, are essential for tumorigenesis and in planta growth by P. syringae pv. savastanoi NCPPB 3335. Front. Plant Sci. 2020;11:1294. https://doi.org/10.3389/fpls.2020.01294.

34. Ma L, Li G. FAR1-RELATED SEQUENCE (FRS) and FRS-RELATED FACTOR (FRF) family proteins in Arabidopsis growth and development. Front Plant Sci. 2018;9:692. https://doi.org/10.3389/fpls.2018.00692.

35. Sotelo-Silveira M, Cucinotta M, Chauvin AL, Chávez Montes RA, Colombo L, Marsch-Martínez N, et al. Cytochrome P450 CYP78A9 is involved in Arabidopsis reproductive development. Plant Physiol. 2013;162(2):779-99. https://doi.org/10.11 04/pp.113.218214.

36. Yonekura-Sakakibara K, Fukushima A, Nakabayashi R, Hanada K, Matsuda F, Sugawara S, et al. Two glycosyltransferases involved in anthocyanin modification delineated by transcriptome independent component analysis in Arabidopsis thaliana. Plant J. 2012;69(1):154-67. https://doi.org/10.1111/j.1365-313X.2011.04779.x.

37. Lu F, Liang X, Lu H, Li Q, Chen Q, Zhang P, et al. Overproduction of superoxide dismutase and catalase confers cassava resistance to Tetranychus cinnabarinus. Sci Rep. 2017;7(1):40179. https://doi.org/10.1038/srep40179.

38. Park CJ, Seo YS. Heat shock proteins: a review of the molecular chaperones for plant immunity. Plant Pathol J. 2015; 31(4):323-33. https://doi.org/10.5423/PPJ.RW.08.2015.0150.

39. Ul Haq S, Khan A, Ali M, Khattak AM, Gai WX, Zhang HX, et al. Heat shock proteins: dynamic biomolecules to counter plant biotic and abiotic stresses. Int J Mol Sci. 2019;20(21):5321.

40. Chen HM, Chang SC, Wu CC, Cuo TS, Wu JS, Juang RH. Regulation of the catalytic behaviour of L-form starch phosphorylase from sweet potato roots by proteolysis. Physiol Plant. 2002;114(4):506-15. https://doi.org/10.1034/j.1399-3 054.2002.1140402.x.

41. Wang Z, Miao H, Liu J, Xu B, Yao X, Xu C, et al. Musa balbisiana genome reveals subgenome evolution and functional divergence. Nat Plants. 2019;5(8):810-21. https://doi.org/10.1038/s41477-019-0452-6.

42. Olsen KM, Wendel JF. A bountiful harvest: genomic insights into crop domestication phenotypes. Annu Rev Plant Biol. 2013;64(1):47-70. https://doi.org/10.1146/annurev-arplant-050312-120048.

43. Salehin M, Bagchi R, Estelle M. SCFTIR1/AFB-based auxin perception: mechanism and role in plant growth and development. Plant Cell. 2015;27(1):9-19. https://doi.org/10.1105/tpc.114.133744.

44. Zhang Y, He P, Ma X, Yang Z, Pang C, Yu J, et al. Auxin-mediated statolith production for root gravitropism. New Phytol. 2019;224(2):761-74. https://doi.org/10.1111/nph.15932.

45. McAdam EL, Meitzel T, Quittenden L, Davidson SE, Dalmais M, Bendahmane Al, et al. Evidence that auxin is required for normal seed size and starch synthesis in pea. New Phytol. 2017;216(1):193-204. https://doi.org/10.1111/nph.14690.

46. Jin Y, Luo Q, Tong H, Wang A, Cheng Z, Tang J, et al. An AT-hook gene is required for palea formation and floral organ number control in rice. Dev Biol. 2011;359(2):277-88. https://doi.org/10.1016/j.ydbio.2011.08.023.

47. Zhao J, Favero DS, Peng H, Neff MM. Arabidopsis thaliana AHL family modulates hypocotyl growth redundantly by interacting with each other via the PPC/DUF296 domain. Proc Natl Acad Sci USA. 2013;110(48):E4688-97. https://doi. org/10.1073/pnas.1219277110

48. Xiao C, Chen F, YU X, Lin C, Fu YF. Over-expression of an AT-hook gene, AHL22, delays flowering and inhibits the elongation of the hypocotyl in Arabidopsis thaliana. Plant Mol Biol. 2009;71(1-2):39-50. https://doi.org/10.1007/s11103-009-9507-9.

49. McCallum EJ, Anjanappa RB, Gruissem W. Tackling agriculturally relevant diseases in the staple crop cassava (Manihot esculenta). Curr Opin Plant Biol. 2017:38:50-8. https://doi.org/10.1016/.pbi.2017.04.008.

50. Kumar K, Purayannur S, Kaladhar VC, Parida SK, Verma PK. mQTL-seq and classical mapping implicates the role of an ATHOOK MOTIF CONTAINING NUCLEAR LOCALIZED (AHL) family gene in Ascochyta blight resistance of chickpea. Plant Cell Environ. 2018;41(9):2128-40. https://doi.org/10.1111/pce.13177. 
51. Baek S, Choi K, Kim GB, Yu HJ, Cho A, Jang H, et al. Draft genome sequence of wild Prunus yedoensis reveals massive inter-specific hybridization between sympatric flowering cherries. Genome Biol. 2018;19(1):127. https://doi.org/10.1186/ s13059-018-1497-y.

52. Zhang J, Zhang X, Tang H, Zhang Q, Hua X, Ma X, et al. Allele-defined genome of the autopolyploid sugarcane Saccharum spontaneum L. Nat Genet. 2018;50(11):1565-73. https://doi.org/10.1038/s41588-018-0237-2.

53. Wu J, Wang Y, Xu J, Korban SS, Fei Z, Tao S, et al. Diversification and independent domestication of Asian and European pears. Genome Biol. 2018;19(1):77. https://doi.org/10.1186/s13059-018-1452-y.

54. Wang M, Zhang L, Zhang Z, Li M, Wang D, Zhang X, et al. Phylogenomics of the genus Populus reveals extensive interspecific gene flow and balancing selection. New Phytol. 2020;225(3):1370-82. https://doi.org/10.1111/nph.16215.

55. Fu YB. Understanding crop genetic diversity under modern plant breeding. Theor Appl Genet. 2015;128(11):2131-42. https://doi.org/10.1007/s00122-015-2585-y.

56. Varshney RK, Saxena RK, Upadhyaya HD, Khan AW, Yu Y, Kim C, et al. Whole-genome resequencing of 292 pigeonpea accessions identifies genomic regions associated with domestication and agronomic traits. Nat Genet. 2017;49(7):10828. https://doi.org/10.1038/ng.3872.

57. Hu W, Ji C, Liang Z, Ye J, Ou W, Ding Z, Zhou G, Tie W, Yan Y, Yang J, Ma L, Yang X, Wei Y, Jin Z, Xie J, Peng M, Wang W, Guo A, Xu B, Guo J, Chen S, Wang M, Zhou Y, Li X, Li R, Xiao X, Wan Z, An F, Zhang J, Leng Q, Li Y, Shi H, Ming R, Li K. Cassava genome resequencing and RNA sequencing. NCBI Sequence Read Archive. https://www.ncbi.nlm.nih.gov/ bioproject/PRJNA578024 (2021).

58. Li H, Durbin R. Fast and accurate short read alignment with Burrows-Wheeler transform. Bioinformatics. 2009;25(14): 1754-60. https://doi.org/10.1093/bioinformatics/btp324.

59. Li H, Handsaker B, Wysoker A, Fennell T, Ruan J, Homer N, et al. The sequence alignment/map format and SAMtools. Bioinformatics. 2009;25(16):2078-9. https://doi.org/10.1093/bioinformatics/btp352.

60. McKenna A, Hanna M, Banks E, Sivachenko A, Cibulskis K, Kernytsky A, et al. The genome analysis toolkit: a MapReduce framework for analyzing next-generation DNA sequencing data. Genome Res. 2010;20(9):1297-303. https://doi.org/10.11 01/gr.107524.110.

61. Cingolani P, Platts A, Wang le L, Coon M, Nguyen T, Wang L, et al. A program for annotating and predicting the effects of single nucleotide polymorphisms, SnpEff: SNPs in the genome of Drosophila melanogaster strain w1118; iso-2; iso-3. Fly (Austin). 2012;6(2):80-92. https://doi.org/10.4161/fly.19695.

62. Tamura K, Peterson D, Peterson N, Stecher G, Nei M, Kumar S. MEGA5: molecular evolutionary genetics analysis using maximum likelihood, evolutionary distance, and maximum parsimony methods. Mol Biol Evol. 2011;28(10):2731-9. https://doi.org/10.1093/molbev/msr121.

63. Letunic I, Bork P. Interactive tree of life (iTOL) v3: an online tool for the display and annotation of phylogenetic and other trees. Nucleic Acids Res. 2016;44(W1):W242-5. https://doi.org/10.1093/nar/gkw290.

64. Purcell S, Neale B, Todd-Brown K, Thomas L, Ferreira MA, Bender D, et al. PLINK: a tool set for whole-genome association and population-based linkage analyses. Am J Hum Genet. 2007;81(3):559-75. https://doi.org/10.1086/519795.

65. Kumar S, Stecher G, Li M, Knyaz C, Tamura K, MEGA X. Molecular evolutionary genetics analysis across computing platforms. Mol Bio Evo. 2018;35(6):1547-9. https://doi.org/10.1093/molbev/msy096.

66. Gu B, Li K, Li Z, Li K. Starch properties of cassava root. Chin J Trop Crops. 2009;30(12):1876-82.

67. Alexander DH, Novembre J, Lange K. Fast model-based estimation of ancestry in unrelated individuals. Genome Res. 2009;19(9):1655-64. https://doi.org/10.1101/gr.094052.109.

68. Hardy OJ, Vekemans X. spagedi: a versatile computer program to analyse spatial genetic structure at the individual or population levels. Mol Ecol Notes. 2002;2(4):618-20. https://doi.org/10.1046/j.1471-8286.2002.00305.X.

69. Fang L, Wang Q, Hu Y, Jia Y, Chen J, Liu B, et al. Genomic analyses in cotton identify signatures of selection and loci associated with fiber quality and yield traits. Nat Genet. 2017;49(7):1089-98. https://doi.org/10.1038/ng.3887.

70. Kim D, Langmead B, Salzberg SL. HISAT: a fast spliced aligner with low memory requirements. Nat Methods. 2015;12(4): 357-60. https://doi.org/10.1038/nmeth.3317.

71. Pertea M, Pertea GM, Antonescu CM, Chang TC, Mendell JT, Salzberg SL. StringTie enables improved reconstruction of a transcriptome from RNA-seq reads. Nat Biotechnol. 2015;33(3):290-5. https://doi.org/10.1038/nbt.3122.

72. Love MI, Huber W, Anders S. Moderated estimation of fold change and dispersion for RNA-seq data with DESeq2. Genome Biol. 2014;15(12):550. https://doi.org/10.1186/s13059-014-0550-8.

73. Pfeifer B, Wittelsbürger U, Ramos-Onsins SE, Lercher MJ. PopGenome: an efficient Swiss army knife for population genomic analyses in R. Mol Biol Evol. 2014;31(7):1929-36. https://doi.org/10.1093/molbev/msu136.

74. Wei Y, Liu W, Hu W, Yan Y, Shi H. The chaperone MeHSP90 recruits MeWRKY20 and MeCatalase1 to regulate drought stress resistance in cassava. New Phytol. 2020;226(2):476-91. https://doi.org/10.1111/nph.16346.

75. Zeng H, Xie Y, Liu G, Wei Y, Hu W, Shi H. Agrobacterium-mediated gene transient overexpression and tobacco rattle virus (TRV)-based gene silencing in cassava. Int J Mol Sci. 2019;20(16):3976. https://doi.org/10.3390/ijms20163976.

\section{Publisher's Note}

Springer Nature remains neutral with regard to jurisdictional claims in published maps and institutional affiliations. 